Pacific Journal of Mathematics

A NOTE OO ABSTRACT MESTEE 


\title{
A NOTE ON ABSTRACT MEASURE
}

\author{
J. M. G. FELL
}

1. Introduction. It has been shown by Segal ([1], our definitions follow [1] whenever possible, except for our introduction of condition (4) into the definition of a measure space) that localizable measure spaces form the largest class of measure spaces to which certain processes of functional analysis may be applied. These measure spaces are characterized as those which are strongly equivalent to direct sums of finite measure spaces. It has some interest to ask under what conditions these finite measures may be obtained as subsets of the original measure space. We shall show that this is always possible provided the 'dimension' of the measure space is equal to or less than the power of the continuum. If one assumes the continuum hypothesis, then every measure space satisfying the given condition on its 'dimension' is localizable in this stronger sense.

2. Definitions. We shall fix a measure space, that is, a triple $M=$ $\langle R, \Re, r\rangle$, where

(1) $\Re$ is a non-void family of subsets of a set $R$, closed under relative complementation and finite union;

(2) $\quad r$ is a nonnegative finite-valued finitely additive function on $\Re$;

(3) $r$ is countably additive in the restricted sense that, if $\left\{E_{n}\right\}$ is a disjoint sequence of sets in $\Re$ such that

$$
\sum_{n=1}^{\infty} r\left(E_{n}\right)=s<\infty,
$$

then

$$
\bigcup_{n=1}^{\infty} E_{n} \in \Re \text { and } r\left(\bigcup_{n=1}^{\infty} E_{n}\right)=s .
$$

We shall also make the following inessential restriction:

(4) If $E \in \Re, r(E)=0, A \subset E$, then $A \in \Re$.

A subset $A$ of $R$ is measurable resp. null if

$$
A \cap E \in \Re \text { resp. } r(A \cap E)=0
$$

for all $E$ in $\Re$. Two subsets $A$ and $B$ of $R$ are equivalent if $A \ominus B(\ominus$ is symmetric difference) is a null set, and almost disjoint if $A \cap B$ is a null set.

The $\sigma$-Boolean algebra of all measurable sets modulo null sets is the measure ring of $M . \quad M$ is localizable if the measure ring of $M$ is complete. By the dimension of $M$ we shall mean the smallest cardinal

Received April 20, 1954. 
number of a maximal almost disjoint subset of $\Re . \quad M$ will be said to be strongly localizable if there exists a (strictly) disjoint subfamily $S$ of $\Re$ such that for every set $A$ in $\Re$, we have

$$
r(A)=\sum_{E \in S} r(A \cap E) .
$$

\section{Results.}

Lemma. If $M$ is strongly localizable, it is localizable.

Proof. Suppose $S$ is a disjoint subfamily of $\Re$ of the kind required in the definition of strong localizability. Consider the measure space $M^{*}=\left\langle R^{*}, \Re^{*}, r^{*}\right\rangle$, where

$$
R^{*}=\bigcup_{E \in S} E,
$$

$\Re^{*}$ is the subfamily of $\Re$ consisting of those $A$ which are contained in a countable union of sets of $S$, and $r^{*}$ is the restriction of $r$ to $\Re^{*}$. Then the measure rings of $M$ and $M^{*}$ are algebraically isomorphic. This follows easily from the facts that every set measurable in $M^{*}$ is also measurable in $M$, and that the complement of $R^{*}$ in $R$ is a null set in $M .^{1)}$

But $M^{*}$ is obviously a direct sum of finite measure spaces [1, Definition 3.1]. Application of Lemma 3.2.2 of [1] now gives the required result.

THeOREM. A measure space $M$ is strongly localizable if either (1) its dimension is equal to or less than the smallest uncountable cardinal, or (2) it is localizable and its dimension is equal to or less than the power of the continum.

Proof. Let $S$ be a maximal almost disjoint subfamily of $\Re$ whose cardinal number is the dimension of $M$. To show $M$ strongly localizable, it is clearly sufficient to show that each $E$ in $S$ can be replaced by an $E^{*}$ in $\Re$ such that (1) $E$ and $E^{*}$ are equivalent, and (2) if $E_{1}$, $E_{2} \in S, E_{1} \neq E_{2}$, then $E_{1}^{*}$ and $E_{2}^{*}$ are disjoint. Let $\alpha$ be the dimension of $M$.

Assume that $\alpha \leqq \boldsymbol{\aleph}_{1}$. The case $\alpha<\boldsymbol{\aleph}_{1}$ is trivial, since then $M$ is $\sigma$-finite, and Corollary 3.2.1 of [1] may be applied directly. Assume $\alpha=$ $\boldsymbol{\mathcal { K }}_{1}$, and let $\left\{E_{\beta}\right\}_{\beta<\Omega}$ be a well-ordering of $S, \Omega$ being the smallest ordinal associated with $\aleph_{1}$. For each $\beta<\Omega$, set

$$
E_{\beta}^{*}=E_{\beta} \sim \bigcup_{\gamma<\beta} E_{\gamma} .
$$

1 These facts clearly require for their proof Condition (4) of the definition of a measure space. 
Since each $\beta<\Omega$ is countable, $E^{*}$ is in $\Re$. The $E^{*}$ are evidently disjoint; and, since the $E_{\beta}$ were almost disjoint, $E_{\beta}$ and $E_{\beta}^{*}$ are equivalent. By the first remark of the proof, the theorem is established for case (1).

Assume now that $M$ is localizable, and that $\alpha \leqq c, c$ being the power of the continuum. Then there is a univalent function $\phi$ on $S$ to the bounded interval $[0,1]$. For each function $f$ in $L^{1}(M)$, we shall define

$$
T(f)=\sum_{E \in S} \phi(E) \int_{H} f(x) d r x .
$$

It is easily verified that $T$ is a continuous linear functional on $L^{1}(M)$. Since $M$ is localizable, the Riesz representation theorem for linear functionals on $L^{1}(M)$ holds [1, Theorem 5.1]; and we obtain a bounded measurable function $g$ such that

$$
T(f)=\int_{R} g(x) f(x) d x \quad \text { for all } f \text { in } L^{1}(M) .
$$

It is evident from the definition of $T$ that, if $E \in S, g(x)=\phi(E)$ for almost all $x$ in $E$. The set

$$
E^{*}=E \cap\{x \mid g(x)=\phi(E)\}
$$

is therefore equivalent to $E$, and for $E, F \in S, E \neq F$, the disjointness of $E^{k}$ and $F^{*}$ is obvious. This completes the proof of the theorem.

If the continuum hypothesis is assumed, the second alternative hypothesis is of no interest. The theorem then states that all measure spaces with dimension equal to or less than $c$ are strongly localizable.

It would be interesting to know whether the assumption about the power of the dimension is necessary for the equivalence of localizability and strong localizability.

\section{REFERENCE}

1. I. E. Segal, “Equivalences of measure spaces”, Amer. J. Math., 73 (1951), 275-313. 



\section{PACIFIC JOURNAL OF MATHEMATICS}

\section{EDITORS}

H. L. Royden

Stanford University

Stanford, California

E. HewitT

University of Washington

Seattle 5 , Washington
R. P. Dilworth

California Institute of Technology Pasadena 4, California

E. G. Straus

University of California

Los Angeles 24, California

\section{ASSOCIATE EDITORS}

E. F. BECKENBACH

C. E. BURGESS

H. BUSEMANN

H. FEDERER

\author{
M. HALL \\ P. R. HALMOS \\ V. GANAPATHY IYER \\ R. D. JAMES
}

M. S. KNEBELMAN

I. NIVEN

T. G. OSTROM

M. M. SCHIFFER
J. J. STOKER

G. SZEKERES

F. WOLF

K. YOSIDA

\section{SUPPORTING INSTITUTIONS}

UNIVERSITY OF BRITISH COLUMBIA

CALIFORNIA INSTITUTE OF TECHNOLOGY

UNIVERSITY OF CALIFORNIA

MONTANA STATE UNIVERSITY

UNIVERSITY OF NEVADA

OREGON STATE COLLEGE

UNIVERSITY OF OREGON

UNIVERSITY OF SOUTHERN CALIFORNIA
STANFORD UNIVERSITY

UNIVERSITY OF UTAH

WASHINGTON STATE COLLEGE

UNIVERSITY OF WASHINGTON

AMERICAN MATHEMATICAL SOCIETY CALIFORNIA RESEARCH CORPORATION HUGHES AIRCRAFT COMPANY 


\section{Pacific Journal of Mathematics}

\section{Vol. 6, No. $1 \quad$ November, 1956}

David Blackwell, An analog of the minimax theorem for vector payoffs..... 1

L. W. Cohen, A non-archimedian measure in the space of real

sequences ..................................... 9

George Bernard Dantzig, Constructive proof of the Min-Max theorem ..... 25

Jim Douglas, On the numerical integration of quasilinear parabolic

differential equations ............................... 35

James Michael Gardner Fell, A note on abstract measure ............. 43

Isidore Isaac Hirschman, Jr., A note on orthogonal systems . . . . . . . . . . 47

Frank Harary, On the number of dissimilar line-subgraphs of a given

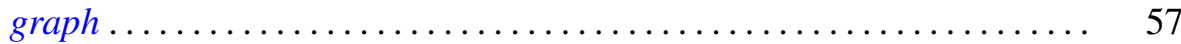

Newton Seymour Hawley, Complex bundles with Abelian group......... 65

Alan Jerome Hoffman, Morris Newman, Ernst Gabor Straus and Olga

Taussky, On the number of absolute points of a correlation ...........

Ernst Gabor Straus and Olga Taussky, Remark on the preceding paper.

Algebraic equations satisfied by roots of natural numbers . . ........ 97

Ralph D. James, Summable trigonometric series ................. 99

Gerald R. Mac Lane, Limits of rational functions . . . . . . . . . . . . . . . 111

F. Oberhettinger, Note on the Lerch zeta function ................. 117

Gerald C. Preston, On locally compact totally disconnected Abelian groups and their character groups ........................... 121

Vikramaditya Singh and W. J. Thron, On the number of singular points, located on the unit circle, of certain functions represented by

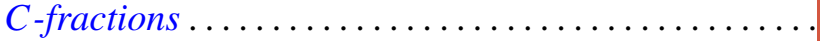

Sherman K. Stein, The symmetry function in a convex body ... 145 Edwin Weiss, Boundedness in topological rings.............

Albert Leon Whiteman, A sum connected with the series for the partition

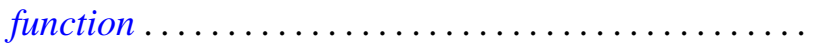

Alfred B. Willcox, Some structure theorems for a class of Banach algebras.

Joseph Lawrence Zemmer, Some remarks on p-rings and their Boolean geometry... 